\title{
Application Value of Dynamic Graphic in Protection of Social Folklore Tradition-Taking Buyi Wedding Series Dynamic Graphics Design as an Example
}

\author{
Honghai Zhang ${ }^{* 1, \text { a }}$, Yongxin Huo ${ }^{1, \mathrm{~b}}$ \\ ${ }^{1}$ School of Design and Arts Beijing Institute of Technology Beijing China
}

\begin{abstract}
With the development of network technology and the change of people's life style, many social folklore traditions in China are gradually forgotten by people. For this reason, protection of social folklore tradition has received much attention in recent years. Based on the analysis of the cultural origin and characteristics of social folklore tradition, this paper proposes to apply the design and expression methods of dynamic graphic to the protection and dissemination of social folklore tradition in order to show them in a more interesting way. Additionally, taking Buyi wedding series dynamic graphic design as an example aims to better explicate the whole design process. This research is conducive to the establishment of social folklore tradition and new media connection, and close to its integration into people's modern life. It can be predicted that the new media based on network technology will become one of the important and efficient means to protect the social folklore tradition.
\end{abstract}

\section{Introduction}

Social folklore tradition has received much attention in recent years because it is associated with Chinese culture, which makes China different. The tradition of social folklore occurs in the process of social adaptation. Festivals, rituals of life and social customs all belong to the tradition of social folklore. [1] As a part of Chinese traditional culture, social folklore tradition has the following characteristics: First of all, because the social folklore tradition is established by Chinese people in the long production and life, it has Chinese national characteristics and a long history. Secondly, China is a multi-ethnic country, each ethnic group has a unique tradition of social folklore. Thirdly, social folklore tradition contains rich Chinese culture.

Compared with the traditional static graphic, the dynamic graphic incorporates new elements, such as dynamic elements, time elements and narrative dimension, which makes the design language more diverse. The principle of human visual cognition shows that human vision is more sensitive to dynamic things than static objects. In other words, when static graphic and dynamic graphic appear in front of people at the same time, dynamic graphic are more attractive and visual impact is stronger.

There has been extensive research regarding dynamic graphic design. However, although it might be feasible to apply dynamic graphics to the protection of social folklore tradition, little attention has been paid. This paper presents a method and the whole process of designing dynamic graphic. Eventually, this combination created an interesting approach in which the social folklore tradition can be spread much more efficiently in the media era.

\section{Dynamic Graphic Design Methods in Social Folklore Tradition}

\subsection{Investigation Process}

The investigation and study of social folklore tradition is the process of collecting and arranging purposeful data after determining the theme of dynamic graphic design. This process is mainly divided into three steps: data collection, field investigation and data collation. The first step, do a good job in the early basic theory and basic data collection. The main contents of this stage include the relevant information of specific social folklore traditions and the relevant theories that may be used. These materials take the form of text, pictures, audio and video, which may come from the Internet, newspapers, books and so on. The collection of relevant data on social folklore tradition can lay a good foundation for field investigation, because understanding the relevant information of the investigation site in advance, such as geographical location, traditional characteristics and living habits, can make a comprehensive plan for field investigation and improve the efficiency of field investigation. The second step, the social folklore tradition carries on the field investigation. Field investigation is a process of testing and applying collected data and theories. Fieldwork can help designers rearrange their understanding of the

a*zhanghonghai@188.com

b2771791241@qq.com 
material and provide an effective reference for visual communication design.[2] Because fieldwork requires investigators to examine specific social folklore traditions in a long and meticulous manner, including processes, utensils, costumes, language, and records of local residents' lifestyles and cultural traditions. In the process of investigation, the investigators will compare this information with the collected information, through theoretical analysis to objectively distinguish all the information, and finally leave the real information related to the subject. The third step, collate all relevant true information. This step is based on the first two steps. It is necessary for researchers to first clarify the theme of the study, then master the real data, and finally select the data that fit the theme of dynamic graphic design and all the research results through the above two steps. And analyze these data to draw further conclusions.

\subsection{Refining of Static Elements}

2.2.1 Generalization of Typical Modeling. The static visual elements in the social folklore tradition are the carriers of culture, such as modeling, color and texture. They are the external manifestations of unique culture and have the uniqueness and representativeness of the specific social folklore tradition. Therefore, the extraction and determination of visual elements help to form a unified design style and enhance the recognition of design objects. As one of the basic static visual elements of social folklore tradition, modeling is mainly derived from the representative types of buildings, costumes and utensils. Because these items and buildings are ubiquitous in life and have become an integral part of social folklore tradition, modeling plays a very important role in the design of dynamic graphics. We should select the most representative or highly relevant version of the theme. The modeling in the previous step should be sorted and processed. According to the theme of dynamic graphic design, these patterns should be classified. At the same time, the artistic expression of deconstruction or reorganization should be used for secondary creation.

2.2.2 Extraction of Symbolic Colors. Compared with modeling, the extraction of color is relatively simple, but in dynamic graphic design, color is also very important for the performance. Just as color is the visual information symbol which is the most sensitive and the fastest to respond to visual stimuli. Colors related to social folklore traditions, i.e., the building in a major ceremony, the apparatus used and the clothing worn. When it comes to a single color, we first think of the hue of color. However, because the use of dynamic graphics to express social folklore traditions is often related to the outline of color and involves a variety of colors. Therefore, in the process of color extraction, color modeling and color combination are equally important. For example, two colors, orange and blue, appear in the picture, different shapes give people different feelings, imagine triangle orange color block and round orange color block, under the action of shape and color, one gives a sharp and delicate feeling, while the other feels lovely and generous. In the process of extracting color elements, after determining the representative color, the most complex step is to formulate the collocation and combination scheme of color. The color scheme is compared with the whole atmosphere of social folklore tradition to find the best color matching scheme with symbolism for dynamic graphic design.

2.2.3 Expression of Unique Texture. In the dynamic graphic design, it is not important to shape and color for the expression of texture, but texture is essential to the expression of social folklore tradition. The articles in life have their own texture, ceramic texture crystal clear, clothing texture soft and comfortable, metal texture is delicate and hard. The texture expression in dynamic graphic can make works carry more information and enrich visual effects. Using dynamic graphic to express the texture of objects can be achieved by color, modeling and texture movement. This requires the design thought to refine the related modelling and the color at the same time must pay attention to grasps its overall characteristic, moreover in the theme expression need situation. According to the above, in the process of refining texture, the texture of objects is put into shape, color and so on, so the extraction of texture is inseparable from the extraction of shape and color. In order to highlight the main information and improve the efficiency of transmission, we can consider abandoning unnecessary texture elements when unnecessary.

\subsection{Transformation of Dynamic Elements}

\subsubsection{Construction of Multidimensional Space. After} refining the static elements, it is necessary to transform them into dynamic graphics. The realization of this transformation mainly starts from three aspects, namely, spatial elements, time elements and sound elements. One of the differences between dynamic graphics and static graphics is that static graphics are presented in a static state on a two-dimensional plane, while dynamic graphics are presented in a moving state on a two-dimensional plane. This difference brings a sense of space to dynamic graphics. The so-called space here refers not to the threedimensional space built by the figure at the end of the motion, but to the sense of space formed on the basis of two-dimensional motion in the process of re-motion, which is a spatial extension on the two-dimensional layout. This kind of spatial feature makes people feel the depth of the picture through the position, size, area, volume and so on.[3] It is precisely because the dynamic graphic can be moved that the speed, direction and track of motion are produced with the motion, which makes the viewer create the illusion of space, thus creating a sense of threedimensional space and making the performance more abundant. In dynamic graphic design, space can be divided into horizontal space and vertical space. The construction of space is directly related to the frequency speed, direction and track of motion, and the frequency speed, direction and track of different motion will produce different semantics. Error or superfluous semantics will 
affect the communication efficiency of social folklore traditional information. This requires designers to understand the relationship between the form and content of dynamic elements, such as high-speed sports create tension, low-speed sports make people feel relatively calm; upward motion means rise, downward motion means decline, moving to the right means forward, moving to the left means backward; straight track makes people feel flat, curve trajectory makes people feel bumpy, point-centered rotation makes people feel dizzy; when curve track with high-speed motion will give people a fast-paced tension. It also includes irregular movements, such as mimicry, imitating existing movements in real life, such as the rolling of small balls, the falling of leaves, the running of animals, and the movement of humans. The form and content of these movements are worthy of careful study and thinking. Designers should also grasp the relationship between static graphic elements, such as modeling, color and texture, and dynamic elements, such as the speed, direction and trajectory of motion. The design of this stage needs to be based on the social custom tradition, to convey the traditional information of social folklore as the ultimate goal, the static graphic elements and dynamic graphic elements are reasonably combined together.

2.3.2 Grasp of Linear Time. In dynamic graphics, because the motion of graphics is a process, it is inevitable to produce motion time. If the motion space transforms the graph from the traditional two-dimensional plane to the virtual three-dimensional plane, then the motion time reflects the motion process of the graph from the traditional single static instantaneous picture to the fixed time. In the design of dynamic graphics, the design of time is mainly reflected in two aspects, first, the order of each element, and then the length of motion time. About the order in which each element appears, space and time are an order between things. Space is used to describe the position and shape of an object, and time is used to describe the order of events.[4] In the tradition of social folklore, such as in a ceremony, its different parts are often interrelated, the former is often the premise of the latter, and the latter is generally the acceptance of the former. If the order between different parts is changed, it will often affect people's understanding and lose its significance and value. The Gestalt principle holds that people will take the initiative to understand the meaning of adjacent visual elements as a whole. In dynamic graphics, the position of visual elements in the picture will affect the viewer's understanding. The length of motion time can be divided into two categories, one is limited motion time, the other is infinite cycle motion time, these two kinds have significant differences in transformation form and function. Limited motion time means that a dynamic graphic work is completed in a fixed time, such as 5 seconds or 10 seconds, with a clear beginning, process, and result, which is used to describe the cause, process, and result of a thing. Therefore, in dynamic graphics, the design of the time length of movement should be properly selected according to the theme, so as to realize the efficient dissemination of information. The grasp of the motion time of dynamic graphics also plays an important role in the expression of the theme in the process of transforming static graphics to dynamic graphics. Effective grasp of time elements will improve the efficiency of social folklore traditional theme expression.

2.3.3 Selection of Auxiliary Sound. The spatial and temporal elements of dynamic graphics mainly transmit information visually, while sound elements transmit information from hearing, realize multi-sensory stimulation to viewers. This is helpful to improve the efficiency of information dissemination and prolong the long-term memory in human brain. The sound elements in dynamic graphics are mainly divided into two types: simulated reality sound and artificial creation sound. The former simulates the sound of nature, that is, by imitating or recording the sound in real life or nature. It is combined with the motion to enhance the reality and vividness of dynamic graphics. The sound elements of simulating reality should be taken from the tradition of social folklore, which is helpful to establish a link with dynamic graphics, in order to enhance the reality and appeal. The artificial sound, refers to the artificial creation of music or songs, this sound applied to dynamic graphics will mainly produce a sense of rhythm and create an atmosphere. Therefore, the control of this music rhythm and the selection of music style is particularly important. There are two main relationships between music rhythm and dynamic graphics, one is dominated by the rhythm of sound, the movement rhythm of dynamic graphics is coordinated with the rhythm of sound. This way gives the viewer a sense of vision and hearing, thus creating a very strong sense of rhythm; the other is dominated by dynamic graphics, ignoring the rhythm of sound. The relationship between music style and dynamic graphics is mainly reflected in whether the design theme is consistent with the style of music. The selection of music style is beneficial to the construction of social folklore traditional atmosphere, so that there is no violation and feeling of separation between music and dynamic graphics. Based on this, sound selection and control are closely related to the other elements of design, so it should run through the whole design process.

\section{Design Case Studies}

\subsection{The Background of the Subject}

Buyi is one of the 56 ethnic minorities in China, mainly distributed in the southwest provinces. It has long history and culture and unique social folklore tradition. Buyi wedding shows the distinctive national characteristics and beliefs. It is different from the traditional Han wedding. Buyi wedding is mainly held in the evening, because the Buyi people regard the night as auspicious time. In the process, there are mainly welcoming, sending relatives, crying relatives, blocking relatives, long table banquet, worship heaven and earth, and so on. In the details, the wedding dress, banquet form and married life are obviously different from Han wedding. Buyi wedding is a Chinese social folklore tradition worth inheriting. 
However, under the impact of the new media era, a large number of Buyi young people have entered the city and accepted a fast-paced lifestyle, so the traditional wedding ceremony has been impacted. Therefore, this design takes the Buyi wedding as the theme and expresses the main process and wedding characteristics through a series of dynamic graphics, in order to protect the social folklore tradition, so as to effectively spread the traditional Chinese folklore culture.

\subsection{Design Process Analysis}

Based on the theoretical research, the design process mainly goes through three stages: Buyi wedding research, static graphic extraction and dynamic graphic transformation.

In the basic research stage, the design theme is the guide, mainly through the reference data and the field investigation, has carried on the thorough and meticulous investigation to the Buyi traditional wedding custom and clothing pattern. Through the material arrangement, Buyi wedding process is divided into 30 small scenes, that is, picking blue, precipitation, weaving, dyeing cloth, drying cloth, dressing up the sedan chair, gong, drum, trumpet, lifting wine, carrying rice, carrying pigs, riding horses, cross-fire basin, bamboo pole dance, wave whistle, blocking relatives, setting off firecrackers, setting off fireworks, fishing, collecting fish, sour soup fish, long table banquet, drinking, heaven and earth, not sitting home, reunion. In addition, the traditional fabric of Buyi is arranged, that is the dress made by embroidery technology, which has rich traditional patterns.

In the static graphic extracting stage, the first is to determine the visual style. By analyzing the traditional pattern on the pick embroidery craft, it is decided to use the similar pattern style to refine and design the static graphic. The character pattern of the groom and bride combined with the dress style at the wedding ceremony, as shown in figure 1.

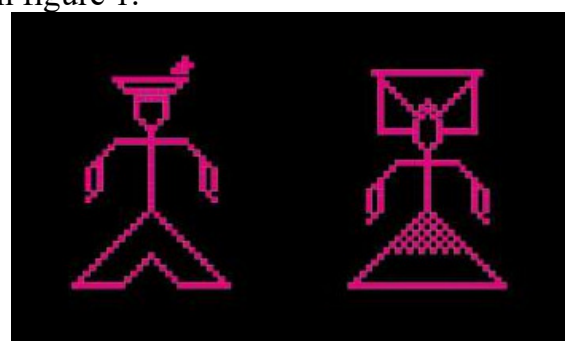

Figure 1: Buyi groom (left) and bride (right)

Then, according to the 30 scene themes defined in the research stage, based on the traditional Buyi embroidery pattern, as shown in figure 2 .

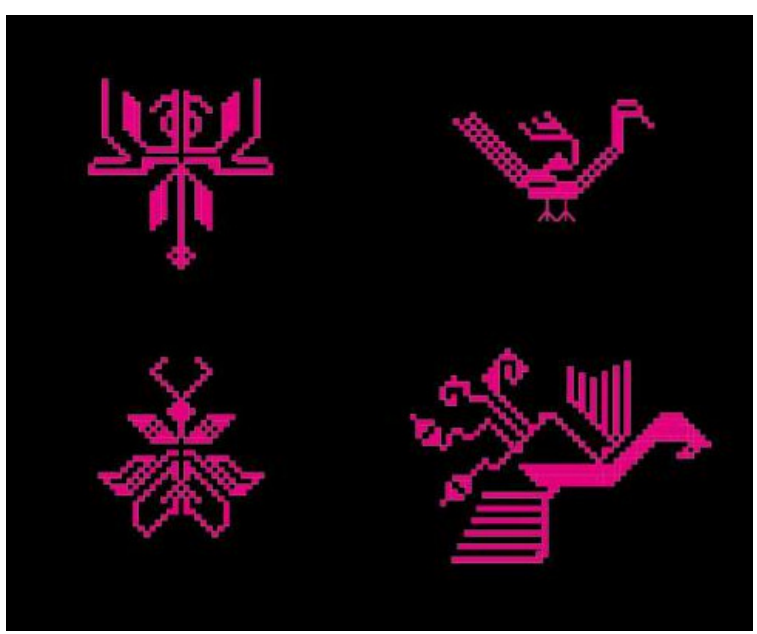

Figure 2: Traditional Buyi embroidery pattern

Finally, the unity of each scene pattern is adjusted. Regarding the color extraction, the pink in the Buyi embroidery pattern is selected to avoid losing the traditional characteristics. The texture mainly manifests the clearance and brightness of the embroidery pattern, as shown in figure 3.

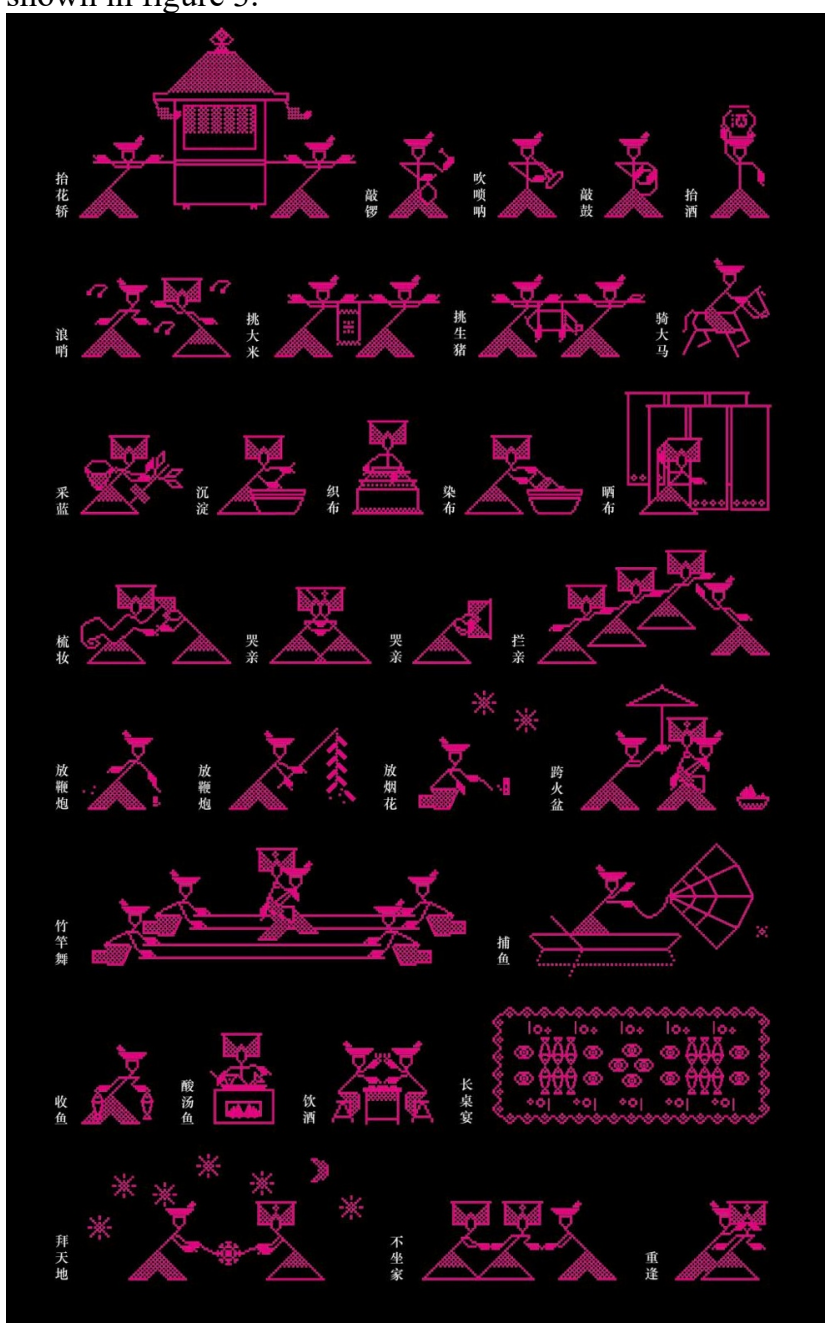

Figure 3: Static graphics of traditional Buyi wedding 


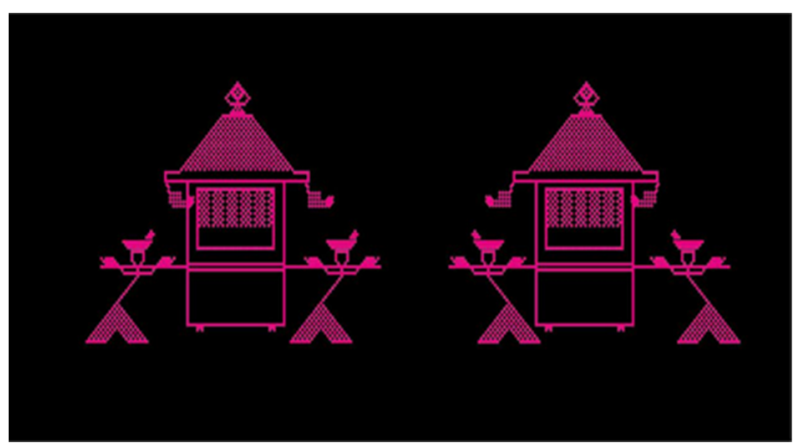

Figure 4: Buyi traditional wedding series dynamic graphics key frame decomposition

In the transformation stage of dynamic graphics. In terms of space construction, as shown in figure 4 , because the pattern on the embroidery fabric belongs to the twodimensional plane, in order to adapt to the increase of popular reading speed in the new media era, we try to refine the movement, control the length of movement, and put the time of each independent small scene in five seconds. In the selection of sound, according to the needs of different scenes, the combination of music and onomatopoeia is adopted. For example, the small scene "reunion" adds lyric music to set off the feelings of nostalgia between husband and wife.

\section{Conclusion}

To sum up, in the new media era, as a part of Chinese traditional culture, social folklore tradition is impacted by people's shallow reading style and fast pace of life. Exploring new methods and measures for the protection of social folklore tradition becomes the need of the times.

This paper explored a new way to apply dynamic graphics to the protection of social folklore tradition. In addition, two problems should be paid attention to in the process of design, the retention of traditional characteristics of social folklore and the relationship between content and form of dynamic elements. It is an important research from a practical point of view because it means that social folklore tradition can be protected in a more interesting, creative and novel way. It is also important from a more theoretical point of view because it confirms the validity of dynamic graphic that could serve as a channel of making the information more attractive and the exchange of information much more efficient.

\section{REFERENCES}

1. Fang Xiao (2019). Folklore Tradition and Rural Revitalization. Journal of Southwest University for Nationalities (Humanities and Social Sciences Edition), 40(05), 28-36.

2. Xiaowei Wang and Rui Huang (2020). Application of Field Investigation in Visual Communication Design. Art Education Research, (17), 68-69.

3. Na An and Jian Liu (2016). The Basic Research of Space and Time: Dynamic Graphics Design. Art Work, (03), 101-103.
4. Hangping Chen and Xuewei Feng (2013). Dynamic Representation of Static Plane Graphics on Screen. Packaging Engineering, 34(12), 25-28. 